\title{
Силлогистика как логика всех отношений между двумя непустыми множествами
}

\author{
Владимир Ильич Маркин \\ МГУ им. М.В. Ломоносова. \\ Российская Федерация, 119991, г. Москва, Ломоносовский пр-т, д. 27, корп. 4. \\ E-mail: markin@philos.msu.ru
}

Аннотация: В работе предлагается язык позитивной силлогистики, алфавит которого содержит все силлогистические константы. Сами эти константы понимаются как знаки отношений между двумя непустыми множествами (объемами двух общих терминов). Среди указанных отношений особое место занимают те, которым соответствует в точности одна из пяти диаграмм Эйлера-Жергонна: (1) равенство множеств, (2) строгое включение первого множества во второе, (3) строгое включение второго множества в первое, (4) перекрещивание множеств, (5) несовместимость множеств. Остальные отношения представляют из себя комбинации эйлеровских. Каждая константа $k$ в предлагаемом силлогистическом языке кодируется последовательностью чисел от 1 до 5 , указывающих на номера тех эйлеровских отношений, при которых простое высказывание формы $S k P$ принимает значение «истина». Например, отношение включения одного непустого множества в другое репрезентирует константа 12 , а отношение совместимости двух непустых множеств - константа 1234. Задается точная теоретико-множественная семантика данного «универсального» языка, соответствующая интуитивному пониманию силлогистических констант. Вводятся понятия выразимости силлогистической константы в «локальном» языке, содержащем лишь некоторые из таких констант, и полноты множества исходных констант некоторого «локального» языка. Константа $k$ выразима в локальном силлогистическом языке с неким набором исходных силлогистических констант, если и только если в этом локальном языке найдется формула $A$, содержащая в точности два термина $S$ и $P$, такая что $A$ эквивалентна формуле $S k P$ в семантике универсального силлогистического языка. Множество силлогистических констант $\left\{k_{1}, k_{2}, \ldots, k_{m}\right\}$ называется полным, если и только если любая силлогистическая константа выразима в языке с исходными силлогистическими константами $k_{1}, k_{2}, \ldots, k_{m}$. В качестве примера доказывается полнота множества исходных констант традиционной силлогистики $(a, e, i, o)$ и множества исходных констант силлогистики Венна (знаков пяти эйлеровских отношений). В универсальном силлогистическом языке строится аксиоматическое исчисление, доказываются метатеоремы о его непротиворечивости и полноте.

Ключевые слова: силлогистика, силлогистическая константа, формализованный язык, семантика, определимость, полная система констант, аксиоматическое исчисление, метатеоремы о непротиворечивости и полноте

Для цитирования: Маркин В.И. Силлогистика как логика всех отношений между двумя непустыми множествами // Логические исследования / Logical Investigations. 2020. T. 26. № 2. C. 39-57. DOI: 10.21146/2074-1472-2020-26-2-39-57

(C) Маркин В.И. 


\section{1. Что такое силлогистическая константа?}

Ассерторическая силлогистика, как известно, является учением о выводах из простых категорических высказываний. Эти высказывания можно считать результатом сочленения двух общих терминов с помощью особого логического термина, который принято называть силлогистической константой. Силлогистическая константа при таком подходе - это единый термин, включающий и внутреннюю связку, и квантор. Указанное единство особенно наглядно в аристотелевской формулировке категорических высказываний, где на первом месте стоит предикат, на втором силлогистическая константа, а в самом конце субъект, например, «P присуще всякому $S », \ll P$ не присуще некоторым $S »$. Схоласты формулировали категорические высказывания иначе, с другой последовательностью терминов: квантор - субъект - внутренняя связка - предикат, например, «Все $S$ есть $P$ », «Некоторые $S$ не есть $P$ ». Но и они при формальной записи этих высказываний (например, $S a P, S o P$ ) соединяли квантор и связку в один логический термин - $a, e, i$ и $о$.

Что же репрезентируют силлогистические константы? При доминирующем в исследованиях по силлогистике экстенсиональном подходе они рассматриваются как знаки отношений между двумя множествами - объемами субъекта и предиката категорического высказывания. Так, константа a обычно трактуется как знак (нестрогого) включения объема субъекта в объем предиката, константа $i$ - как знак совместимости этих объемов (наличия в данных множествах общих элементов), константа $e-$ как знак несовместимости указанных множеств, константа $o$ - как знак невключения первого объема во второй.

В качестве семантического инструмента в силлогистике широко используются круговые диаграммы:
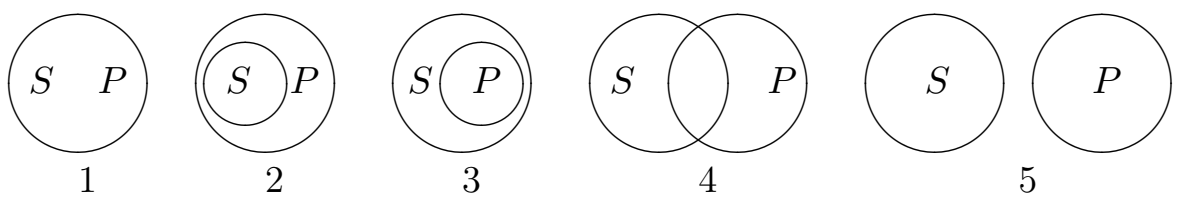

5

Рис. 1. Диаграммы Эйлера-Жергонна

Каждая из этих диаграмм изображает определенное отношение между двумя множествами, которые являются объемами общих терминов $S$ и $P$ :

1) равенство объемов $S$ и $P$;

2) строгое включение объема $S$ в объем $P$; 
3) строгое включение объема $P$ в объем $S$;

4) перекрещивание объемов $S$ и $P$;

5) несовместимость (внеположенность) объемов $S$ и $P$.

При этом предполагается, что оба эти множества непусты. Будем называть указанные пять отношений «эйлеровскими».

Нетрудно заметить, что лишь одна стандартная силлогистическая константа из четырех (а именно $e$ ) репрезентирует эйлеровское отношение (отношение 5). Высказывание формы $S e P$ истинно лишь в том случае, когда объемы $S$ и $P$ не имеют общих элементов. При всех иных эйлеровских отношениях между этими (непустыми) множествами $S e P$ оказывается ложным. Что касается других стандартных силлогистических констант ( $a, i$ и $o$ ), то категорические высказывания, образованные с их помощью, истинны при нескольких различных эйлеровских отношениях между объемами субъекта и предиката: $S a P$ истинно при отношениях $1-2, S i P$ - при отношениях $1-4$, SoP - при отношениях $3-5$.

В истории логики предпринимались попытки построения силлогистических теорий с нестандартными исходными наборами силлогистических констант. Так, Н.А. Васильев Васильев, 1910 предложил использовать вместо неопределенно-частных высказываний $(\mathrm{SiP}$ и $\mathrm{SoP}$ ) определенночастное: «Только некоторые $S$ есть $P$ ». Оно принимает значение «истина», е.т.е. (если и только если) объемы $S$ и $P$ находятся в отношениях 3 или 4.

Дж. Венн [Venn, 1881] предложил систему силлогистического типа с пятью исходными видами категорических высказываний: четырьмя утвердительными и общеотрицательным. При выделении четырех разновидностей утвердительных высказываний он опирался на классификацию суждений У. Гамильтона, который указывал на необходимость учета квантификации не только субъекта, но и предиката. При таком подходе утвердительные высказывания можно разделить на обще-общие («Все $S$ есть все $P »)$, обще-частные («Все $S$ есть некоторые $P$ »), частно-общие («Некоторые $S$ есть все $P \gg)$ и частно-частные («Некоторые $S$ есть некоторые $P \gg)$.

Высказывания каждого из пяти видов логики Венна истинны ровно при одном эйлеровском отношении между объемами $S$ и $P$ : общеотрицательные, как уже говорилось, при отношении 5 , а что касается утвердительных, то обще-общие истинны при отношении 1 , обще-частные при отношении 2 , частно-общие при отношении 3 , частно-частные при отношении 4. Для каждого из перечисленных видов высказываний можно ввести свою собственную силлогистическую константу, репрезентирующую определенное эйлеровское отношение между двумя непустыми множествами. 
Выше уже отмечалось, что кроме эйлеровских имеются и иные бинарные отношения между непустыми множествами: отношение нестрогого включения, соответствующее константе $a$, отношение объемной совместимости, соответствующее константе $i$, отношение, фиксирующееся в истинном определенно-частном высказывании, и др. Очевидно, что число всех возможных отношений между двумя непустыми множествами равно мощности множества всех подмножеств $\{1,2,3,4,5\}$, т. е. тридцати двум. Каждому из этих отношений можно сопоставить свою уникальную силлогистическую константу.

Удобно в запись произвольной силлогистической константы $k$ включать номера тех эйлеровских отношений, при которых категорическое высказывание формы $S k P$ истинно.

Запись констант, репрезентирующих сами эйлеровские отношения, будет включать одно число (от 1 до 5), которое совпадает с номером соответствующего отношения. Тогда, например, формой обще-общих утвердительных высказываний будет выражение $S 1 P$, а формой общеотрицательных высказываний - выражение $S 5 P$.

Константы, репрезентирующие 26 неэйлеровских отношений между двумя непустыми множествами, будут представлять собой многокомпонентные последовательности чисел. Например, константа а будет записываться как 12 (а форма высказывания «Все $S$ есть $P$ как $S 12 P$ ), константа $i$ как 1234, константа $о$ как 345, а константа, образующая определенночастное высказывание, как 34. Договоримся располагать числа в составе многокомпонентных констант в порядке их возрастания.

Еще одна константа, которая выделяется с целью общности рассмотрения, является нулъкомпонентной. Она репрезентирует «пустое» отношение, т. е. такое отношение, в котором не находятся никакие два непустых множества. Форма высказывания, образованного с помощью нулькомпонентной константы, будет фиксироваться выражением $S P$.

Другим предельным случаем отношения между двумя непустыми множествами является «универсальное» отношение, т. е. отношение, в котором находятся любые два таких множества. Данное отношение репрезентируется константой $12345^{1}$.

Таким образом, силлогистической константой назовем последовательность (возможно пустую) $x_{1} x_{2} \ldots x_{n}$ попарно различных чисел из множества $\{1,2,3,4,5\}$, расположенных по возрастанию.

\footnotetext{
${ }^{1}$ Нулькомпонентная константа и константа 12345 яваляются, по сути, силлогистическими аналогами пропозициональных констант ложности и истинности, соответственно. Как будет показано ниже, $S P$ является формой логически ложного , а $S 12345 P$ формой логически истинного простого высказывания.
} 
Учитывая, что за каждым числом $x_{i}$ в составе некоторой силлогистической константы $k$ стоит определенная круговая диаграмма, числовой код $x_{1} x_{2} \ldots x_{n}$ задает полный список эйлеровских отношений, при которых высказывание формы $S k P$ принимает значение «истина». А сама константа $k$ с семантической точки зрения репрезентирует такое отношение между объемами терминов $S$ и $P$, которое является объединением эйлеровских отношений, кодируемых числами $x_{1}, x_{2}, \ldots, x_{n}$.

\section{2. Универсальный язык позитивной силлогистики и его семантика}

Построим позитивную силлогистику, язык которой содержит тридцать две силлогистические константы - знаки всех возможных бинарных отношений между непустыми множествами.

С использованием данного «универсалъного» силлогистического языка и логики, в нем построенной, может быть развита общая метатеория силлогистических констант позитивной силлогистики. В ее рамках можно выделять различные свойства этих констант и отношения между ними. Появляется возможность ввести точное понятие выразимости (определимости) силлогистической константы в «локалъном» силлогистическом языке, содержащем не все силлогистические константы. Также могут быть исследованы различные характеристики множеств (наборов) силлогистических констант, в том числе введено понятие полного множества констант и сформулирована задача по установлению критериев полноты.

Итак, под универсальным силлогистическим языком будем понимать язык позитивной силлогистики, алфавит которого содержит все силлогистические константы. Помимо них в алфавит включаются бесконечный список общих терминов, пропозициональные связки и скобки. Атомарные формулы универсального силлогистического языка имеют вид $S k P$, где $S$ и $P$ - общие термины, а $k$ - произвольная силлогистическая константа (если константа $k$ представляет собой пустую последовательность, то $S k P$ записывается как $S P$ ). Сложные формулы образуются обычным способом с помощью пропозициональных связок. Будем обозначать множество всех формул данного языка как $\mathbf{L}_{\mathbf{U}}$.

Сформулируем точную семантику универсального силлогистического языка, основываясь на содержательных интуициях, изложенных в предыдущем разделе.

Моделью назовем пару $\langle\mathbf{D}, \varphi\rangle$, где $\mathbf{D}$ - произвольное непустое множество, $\varphi-$ функция, сопоставляющая каждому общему термину некоторое непустое подмножество D: $\varphi(S) \subseteq \mathbf{D}$ и $\varphi(S) \neq \varnothing$. 
Определим понятие значимости (истинности) формулы $A \in \mathbf{L}_{\mathbf{U}}$ в модели $\langle\mathbf{D}, \varphi\rangle$. С этой целью введем трехместный предикат $\mathcal{V} . \mathcal{V}(A, \mathbf{D}, \varphi)$ читается так: «формула $A$ значима в модели $\langle\mathbf{D}, \varphi\rangle »$.

Зададим сначала условия значимости атомарных формул, образованных посредством нулькомпонентной константы:

$\mathcal{V}_{\varnothing}$. Неверно, что $\mathcal{V}(S P, \mathbf{D}, \varphi)$

(т. е. формулы вида $S P$ не являются значимыми ни в какой модели).

Зададим далее условия значимости атомарных формул, образованных посредством однокомпонентных силлогистических констант:

$\mathcal{V}_{1} \cdot \mathcal{V}(S 1 P, \mathbf{D}, \varphi)$, е.т.е. $\varphi(S)=\varphi(P)$

$\mathcal{V}_{2} . \mathcal{V}(S 2 P, \mathbf{D}, \varphi)$, е.т.е. $\varphi(S) \subset \varphi(P)$;

$\mathcal{V}_{3} . \mathcal{V}(S 3 P, \mathbf{D}, \varphi)$, е.т.е. $\varphi(P) \subset \varphi(S)$;

$\mathcal{V}_{4}$. $\mathcal{V}(S 4 P, \mathbf{D}, \varphi)$, е.т.е. $\varphi(S) \cap \varphi(P) \neq \varnothing$ и $\varphi(S) \backslash \varphi(P) \neq \varnothing$ и $\varphi(P) \backslash \varphi(S) \neq \varnothing$

$\mathcal{V}_{5} \cdot \mathcal{V}(S 5 P, \mathbf{D}, \varphi)$, е.т.е. $\varphi(S) \cap \varphi(P)=\varnothing$

$\left(\mathcal{V}_{1}-\mathcal{V}_{5}\right.$ фиксируют в теоретико-множественном языке те же самые эйлеровские отношения, которые изображены на круговых диаграммах 1-5).

Зададим наконец условия значимости атомарных формул, образованных посредством многокомпонентных силлогистических констант. Пусть силлогистическая константа $k$ представляет собой последовательность $x_{1} x_{2} \ldots x_{n}$ попарно различных чисел из множества $\{1,2,3,4,5\}$, расположенных по возрастанию, причем $2 \leqslant n \leqslant 5$.

$\mathcal{V}_{M} \cdot \mathcal{V}\left(S x_{1} x_{2} \ldots x_{n} P, \mathbf{D}, \varphi\right)$, е.т.е. $\mathcal{V}\left(S x_{1} P, \mathbf{D}, \varphi\right)$ или $\mathcal{V}\left(S x_{2} P, \mathbf{D}, \varphi\right)$ или ... или $\mathcal{V}\left(S x_{n} P, \mathbf{D}, \varphi\right)$

(формула, образованная с помощью многокомпонентной константы, значима в некоторой модели, е.т.е. в этой модели значима хотя бы одна формула, образованная с помощью однокомпонентной константы, входящей в данную многокомпонентную константу).

$\mathcal{V}_{C}$. Условия значимости сложных формул стандартные.

Формула $A \in \mathbf{L}_{\mathbf{U}}$ общезначима в семантике универсального силлогистического языка (U-общезначима), е.т.е. $\mathcal{V}(A, \mathbf{D}, \varphi)$ в любой модели $\langle\mathbf{D}, \varphi\rangle$.

Формула $A \in \mathbf{L}_{\mathbf{U}}$ выполнима в семантике универсального силлогистического языка (U-выполнима), е.т.е. $\mathcal{V}(A, \mathbf{D}, \varphi)$ по крайней мере в одной модели $\langle\mathbf{D}, \varphi\rangle$.

Из сформулированных выше условий значимости вытекает, что формула вида $S 12345 P$ общезначима в данной семантике, а формула вида $S P$ не является выполнимой. 
Верным является также следующее утверждение: если силлогистическая константа $k$ содержит число 1 , то формула $S k S$ общезначима в семантике универсального силлогистического языка; в противном случае формула $S k S$ не является выполнимой. Заметим, что известные законы силлогистического тождества $S a S$ и $S i S$ являются частными случаями общезначимых формул вида $S k S$, где константа $k$ содержит 1 , ведь $a$ в нашей записи - это 12 , а $i-1234$.

В построенной логике имеют место обобщения других известных законов традиционной силлогистики: силлогистических вариантов законов противоположностей (напр., $\neg(S a P \wedge S e P))$ и законов исключенного третьего (напр., $S i P \vee S o P$ ). Если константы $k_{1}$ и $k_{2}$ не содержат в своем составе ни одного одинакового числа, то формула $S k_{1} P \wedge S k_{2} P$ не является выполнимой (а значит $\neg\left(S k_{1} P \wedge S k_{2} P\right)$ общезначима). Если каждое число из множества $\{1,2,3,4,5\}$ входит в состав хотя бы одной из констант $k_{1}$ или $k_{2}$, то формула $S k_{1} P \vee S k_{2} P$ общезначима.

Формулы $A$ и $B$ эквивалентны в семантике универсального силлогистического языка, е.т.е. для любой модели $\langle\mathbf{D}, \varphi\rangle$ верно, что $\mathcal{V}(A, \mathbf{D}, \varphi)$, е.т.е. $\mathcal{V}(B, \mathbf{D}, \varphi)$.

Из $\mathcal{V}_{M}$ и $\mathcal{V}_{C}$ следует, что формула $S x_{1} x_{2} \ldots x_{n} P$ эквивалентна формуле $S x_{1} P \vee S x_{2} P \vee \ldots \vee S x_{n} P$.

Можно сформулировать принцип установления отношения противоречия между атомарными формулами с одинаковыми субъектами и предикатами: если константы $k_{1}$ и $k_{2}$ не содержат в своем составе ни одного одинакового числа и каждое число из множества $\{1,2,3,4,5\}$ входит в состав хотя бы одной из этих констант, то формула $S k_{1} P$ эквивалентна формуле $\neg S k_{2} P$ и протворечит формуле $S k_{2} P$. Частным случаем этого принципа являются законы диагоналей логического квадрата: $\operatorname{SaP}$ (т. е. $S 12 P$ ) противоречит $S o P$ (т. е. $S 345 P$ ), а $S e P$ (т. е. $S 5 P$ ) противоречит $\operatorname{SiP}($ т. е. $S 1234 P$ ).

В рамках данной силлогистики можно развить интересное учение об обращении. Приведем некоторые результаты, связанные с данным вопросом. Если силлогистическая константа $k$ не содержит чисел 2 и 3 или же содержит как число 2 , так и число 3 , то формула $S k P$ эквивалентна формуле $P k S$. Если силлогистическая константа $k_{1}$ содержит число 2 , но не содержит число 3 , а константа $k_{2}$ получается из $k_{1}$ заменой 2 на 3 , то формула $S k_{1} P$ эквивалентна формуле $P k_{2} S$. Если силлогистическая константа $k_{1}$ содержит число 3 , но не содержит число 2 , а константа $k_{2}$ получается из $k_{1}$ заменой 3 на 2 , то формула $S k_{1} P$ эквивалентна формуле $P k_{2} S$.

Докажем метатеорему об эквивалентной замене. 
Теорема 1. Пусть $A$ и $B-$ произвольные формульи из $\mathbf{L}_{\mathbf{U}}, C_{A}-\oint о р$ мула данного языка с выделенным вхождением подформулы $A$, а $C_{B}-$ формула, получающался в результате замены выделенного вхождения $A$ в $C_{A}$ на формулу B. Если формулы $A$ и $B$ эквивалентны в семантике универсального силлогистического языка, то формулы $C_{A}$ и $C_{B}$ также эквивалентны в этой семантике.

Доказательство. Доказательство ведется индукцией по числу пропозициональных связок в формуле $C_{A}$.

Базис индукции. Пусть $C_{A}$ не содержит пропозициональных связок, т. е. это атомарная формула. Тогда $C_{A}=A$, а $C_{B}=B$. Поэтому если $A$ и $B$ эквивалентны в семантике универсального силлогистического языка, то $C_{A}$ и $C_{B}$ также эквивалентны в ней.

Индуктивный переход. Допустим, что теорема верна для любой формулы с меньшим, чем у $C_{A}$, числом пропозициональных связок.

Пусть главным знаком в формуле $C_{A}$ является отрицание, тогда $C_{A}$ имеет вид $\neg D_{A}$, а $C_{B}$ имеет вид $\neg D_{B}$. Формула $D_{A}$ имеет меньше пропозициональных связок, чем $C_{A}$, поэтому, по индуктивному допущению, если $A$ эквивалентна $B$, то $D_{A}$ эквивалентна $D_{B}$. А из последнего, в силу семантики пропозиционального отрицания, вытекает, что формула $\neg D_{A}$ эквивалентна формуле $\neg D_{B}$.

Пусть главным знаком в формуле $C_{A}$ является конъюнкция, тогда $C_{A}$ имеет вид $D_{A} \wedge E$ либо $D \wedge E_{A}$. В первом случае формула $C_{B}$ имеет вид $D_{B} \wedge E$, во втором - $D \wedge E_{B}$. Рассмотрим первый случай (второй аналогичен). Формула $D_{A}$ имеет меньше пропозициональных связок, чем $C_{A}$, поэтому, по индуктивному допущению, если $A$ эквивалентна $B$, то $D_{A}$ эквивалентна $D_{B}$. В силу условий значимости конъюнктивных формул, если $D_{A}$ эквивалентна $D_{B}$, то $D_{A} \wedge E$ (т. е. $C_{A}$ ) эквивалентна $D_{B} \wedge E$ (т. е. $\left.C_{B}\right)$. По транзитивности имеем: если $A$ эквивалентна $B$, то $C_{A}$ эквивалентна $C_{B}$.

$\mathrm{B}$ случаях, когда главным знаком формулы $C_{A}$ являются иные пропозициональные связки, рассуждение осуществляется аналогично.

\section{3. Локальные силлогистические языки и полные множества силлогистических констант}

Обычно в язык позитивной силлогистики в качестве исходных вводят не все, а только часть силлогистических констант. Такие языки будем называть «локальными». K числу локальных относятся языки традиционной силлогистики, силлогистик Венна и Васильева.

При семантическом построении силлогистики в локальном языке исходным константам сопоставляются некоторые отношения между объемами двух общих терминов (мы будем рассматривать лишь случай, когда эти 
термины непусты). Предполагается, однако, что этих отношений достаточно для того, чтобы выразить и все другие бинарные объемные отношения. В таком случае можно считать множество исходных констант полным.

Используя семантику универсального силлогистического языка, введем точные определения выразимости константы в локальном языке и полноты множества силлогистических констант.

Пусть $\left\{k_{1}, k_{2}, \ldots, k_{m}\right\}$ - множество исходных силлогистических констант некоторого локального языка. Каждая константа $k_{j}$ из этого множества записывается с использованием числового кода, о котором шла речь в предыдущем разделе, т. е. основываясь на семантике этого языка, мы в самой записи $k_{j}$ фиксируем те эйлеровские отношения между объемами $S$ и $P$, при которых высказывание формы $S k_{j} P$ истинно.

Константа $k$ выразима в локальном силлогистическом языке с исходными силлогистическими константами $k_{1}, k_{2}, \ldots, k_{m}$, е.т.е. в этом локальном языке найдется формула $A$, содержащая в точности два термина $S$ и $P$, такая что $A$ эквивалентна формуле $S k P$ в семантике универсального силлогистического языка.

Например, константа 12 (т. е. а) выразима в языке силлогистики Венна с исходными константами $1,2,3,4,5$, поскольку в этом языке найдется формула, а именно $S 1 P \vee S 2 P$, которая содержит термины $S$ и $P$ и эквивалентна в семантике универсального языка формуле $S 12 P$.

Другой пример. Константа 1 выразима в языке с исходными константами $12,1234,345,5$ (они соответствуют стандартным константам традиционной силлогистики $a, i, o, e)$, так как в этом языке найдется формула, а именно $S 12 P \wedge P 12 S$, которая содержит термины $S$ и $P$ и эквивалентна в семантике универсального языка формуле $S 1 P$.

Множество силлогистических констант $\left\{k_{1}, k_{2}, \ldots, k_{m}\right\}$ называется nолным, е.т.е. любая силлогистическая константа выразима в языке с исходными силлогистическими константами $k_{1}, k_{2}, \ldots, k_{m}$.

Рассмотрим два локальных языка (язык силлогистики Венна и язык традиционной силлогистики) и докажем полноту множеств их исходных констант. Пусть $\mathbf{L}_{\mathbf{V}}$ - множество формул языка силлогистики Венна, a $\mathbf{L}_{\mathbf{T}}$ - множество формул языка традиционной силлогистики.

Теорема 2. Множество исходнъх констант силлогистики Венна $\{1,2,3,4,5\}$ является полным.

Доказательство. При семантическом построении силлогистики Венна используем модели того же типа, что и в семантике универсального силлогистического языка. В данном локальном языке имеется всего пять видов 
атомарных формул. Условия значимости для них - это $\mathcal{V}_{1}-\mathcal{V}_{5}$. Для определения условий значимости сложных формул используем $\mathcal{V}_{C} . \mathbf{V}$-общезначимой назовем формулу $A \in \mathbf{L}_{\mathbf{V}}$, значимую в любой модели при $\mathcal{V}$, включающем $\mathcal{V}_{1}-\mathcal{V}_{5}, \mathcal{V}_{C}$.

Нужно показать, что все тридцать две силлогистические константы универсального языка выразимы в рассматриваемом локальном языке. Эти константы, как уже говорилось, бывают однокомпонентными, многокомпонентными и нулькомпонентными.

Все однокомпонентные константы входят в множество исходных констант локального языка, а условия значимости атомарных формул с этими константами в семантиках универсального и локального языков совпадают. Поэтому все они выразимы в данном локальном языке.

Произвольная многокомпонентная константа $x_{1} x_{2} \ldots x_{n}$ также выразима в нем, поскольку в семантике универсального языка формуле $S x_{1} x_{2} \ldots x_{n} P$ эквивалентна формула $S x_{1} P \vee S x_{2} P \vee \ldots \vee S x_{n} P$, содержащая в точности два общих термина $S$ и $P$.

Нулькомпонентная силлогистическая константа также является выразимой: формуле $S P$ в семантике универсального языка эквивалентна любая невыполнимая формула локального языка, содержащая лишь термины $S$ и $P$, например, формула $S 2 S \wedge P 2 P$.

Теорема 2 дает более простой критерий полноты произвольного множества силлогистических констант: множество констант $\left\{k_{1}, k_{2}, \ldots, k_{m}\right\}$ является полным, е.т.е. в языке с исходными константами $k_{1}, k_{2}, \ldots, k_{m}$ выразимы константы $1,2,3,4,5$.

Будем использовать этот критерий при доказательстве следующей метатеоремы.

Теорема 3. Множсество исходных констант традиционной силлогистики $\{a$, е,$i$, о $\}$ является полным.

Доказателъство. Семантика традиционной позитивной силлогистики обычно строится следующим образом. Используется тот же класс моделей $\langle\mathbf{D}, \varphi\rangle$, где $\mathbf{D} \neq \varnothing, \varphi(S) \subseteq \mathbf{D}$ и $\varphi(S) \neq \varnothing$. Условия значимости четырех типов атомарных формул определяются так:

$\mathcal{V}_{a} . \mathcal{V}(S a P, \mathbf{D}, \varphi)$, е.т.е. $\varphi(S) \subseteq \varphi(P)$;

$\mathcal{V}_{e} . \mathcal{V}(S e P, \mathbf{D}, \varphi)$, е.т.е. $\varphi(S) \cap \varphi(P)=\varnothing$;

$\mathcal{V}_{i}$. $\mathcal{V}($ SiP $, \mathbf{D}, \varphi)$, е.т.е. $\varphi(S) \cap \varphi(P) \neq \varnothing$;

$\mathcal{V}_{o} . \mathcal{V}(S o P, \mathbf{D}, \varphi)$, е.т.е. $\varphi(S) \backslash \varphi(P) \neq \varnothing$.

Для сложных формул принимаются стандартные условия значимости $\left(\mathcal{V}_{C}\right)$. $\mathbf{T}$-общезначимой назовем формулу $A \in \mathbf{L}_{\mathbf{T}}$, значимую в любой модели при $\mathcal{V}$, включающем $\mathcal{V}_{a}, \mathcal{V}_{e}, \mathcal{V}_{i}, \mathcal{V}_{o}, \mathcal{V}_{C}$ 
Покажем сначала, что каждую исходную константу традиционной силлогистики можно эквивалентным образом заменить некоторой константой универсального силлогистического языка.

$\mathcal{V}(S a P, \mathbf{D}, \varphi)$, е.т.е. (в силу $\left.\mathcal{V}_{a}\right) \varphi(S) \subseteq \varphi(P)$, е.т.е (в силу связи отношений нестрогого включения, равенства множеств и строгого включения) $\varphi(S)=\varphi(P)$ или $\varphi(S) \subset \varphi(P)$, е.т.е. (в силу $\mathcal{V}_{1}$ и $\left.\mathcal{V}_{2}\right) \mathcal{V}(S 1 P, \mathbf{D}, \varphi)$ или $\mathcal{V}(S 2 P, \mathbf{D}, \varphi)$, е.т.е. (в силу $\left.\mathcal{V}_{M}\right) \mathcal{V}(S 12 P, \mathbf{D}, \varphi)$.

Таким образом, константа $а$ есть та же константа, что и 12.

$\mathcal{V}(S e P, \mathbf{D}, \varphi)$, е.т.е. (в силу $\left.\mathcal{V}_{e}\right) \varphi(S) \cap \varphi(P)=\varnothing$, е.т.е. (в силу $\mathcal{V}_{5}$ ) $\mathcal{V}(S 5 P, \mathbf{D}, \varphi)$.

Таким образом, константа $e$ есть та же константа, что и 5.

В семантике традиционной силлогистики условия значимости SiP противоречат условиям значимости $S e P$, а условия значимости $S o P$ противоречат условиям значимости $S a P$. Согласно сформулированному в предыдущем разделе принципу установления отношения противоречия между атомарными формулами с одинаковыми субъектами и предикатами, формуле $S 5 P$ в семантике универсального силлогистического языка противоречит формула $S 1234 P$, а формуле $S 12 P$ - формула $S 345 P$. Поэтому $i$ есть та же константа, что и 1234, а $о$ - та же константа, что и 345.

Остается показать, что константы $1,2,3,4,5$ выразимы в языке традиционной силлогистики. Для этого для каждой атомарной формулы $S k P \in \mathbf{L}_{\mathbf{V}}$ укажем формулу из $\mathbf{L}_{\mathbf{T}}$, эквивалентную ей в семантике универсального силлогистического языка и содержащую два термина $S$ и $P$.

Формуле $S 1 P$ в семантике универсального силлогистического языка эквивалентна формула $S 12 P \wedge P 12 S$, т. е. $S a P \wedge P a S$.

Формуле $S 2 P$ в семантике универсального языка эквивалентна формула $S 12 P \wedge P 345 S$, т. е. $S a P \wedge P o S$.

Формуле $S 3 P$ в семантике универсального силлогистического языка эквивалентна формула $S 345 P \wedge P 12 S$, т. е. $S o P \wedge P a S$.

Формуле $S 4 P$ в семантике универсального силлогистического языка эквивалентна формула $S 1234 P \wedge S 345 P \wedge P 345 S$, т. е. $S i P \wedge S o P \wedge P o S$.

Формула $S 5 P$ в семантике универсального силлогистического языка эквивалентна самой себе, т. е. $S e P$.

Демонстрация справедливости этих утверждений не представляет сложности и основана исключительно на условиях значимости формул в семантике универсального силлогистического языка.

Нередки ситуации, когда некоторые константы в полном множестве силлогистических констант оказываются излишними, поскольку выразимы посредством других. Поэтому важным является понятие минимально полного множества констант. 
Множество силлогистических констант $\left\{k_{1}, k_{2}, \ldots, k_{m}\right\}$ называется минимально полным, е.т.е. оно является полным, и любое правильное подмножество данного множества не является полным.

Множество исходных констант традиционной силлогистики $\{12,1234$, $345,5\}$ не является минимально полным, поскольку, например, константы 1234 и 345 выразимы в языке с исходными константами 12 и 5: формула $S 1234 P$ эквивалентна $\neg S 5 P$, а формула $S 345 P$ эквивалентна $\neg S 12 P$. Множество констант $\{12,5\}$, а также множества $\{12,1234\},\{345,5\}$ и $\{1234$, $345\}$ являются минимально полными.

Множество исходных констант силлогистики Венна $\{1,2,3,4,5\}$ также не относится к числу минимально полных. Так, например, константы 3 и 4 выразимы в языке с более узким множеством исходных констант $\{1,2,5\}$ : формула $S 3 P$ эквивалентна формуле $P 2 S$, а формула $S 4 P$ эквивалентна формуле $\neg S 1 P \wedge \neg S 2 P \wedge \neg P 2 S \wedge \neg S 5 P$.

\section{4. Формализация «универсальной» позитивной силлогистики}

Традиционный вариант позитивной силлогистики (множество Т-общезначимых формул) формализует широко известная силлогистика Я. Лукасевича [Лукасевич, 1959] - аксиоматическое исчисление С4 по классификации В.А. Смирнова [Смирнов, 2002].

Утверждение 1. Для любой формуль $A \in \mathbf{L}_{\mathbf{T}}$ верно, что $A$ доказуема в исчислении $\mathbf{C 4}$, е.т.е. $A$ является Т-общезначимой (см., напр., Мчедлишвили, 1985|).

С целью формализации силлогистики Венна в Дубаков, Маркин, 2007 было предложено исчисление $\mathbf{C 4 V}$, схемами аксиом которого являются:

V0. Пропозициональные тавтологии;
V1. $(M 1 P \wedge S 1 M) \supset S 1 P$;
V11. $S 5 P \supset P 5 S$;
V2. $(M 1 P \wedge S 2 M) \supset S 2 P$;
V12. $S 1 S$;
V3. $(M 2 P \wedge S 1 M) \supset S 2 P$;
V13. $\neg(S 1 P \wedge S 2 P)$;
V4. $(M 2 P \wedge S 2 M) \supset S 2 P$;
V14. $\neg(S 1 P \wedge S 3 P)$;
V5. $(M 5 P \wedge S 1 M) \supset S 5 P$;
V15. $\neg(S 1 P \wedge S 4 P)$;
V6. $(M 5 P \wedge S 2 M) \supset S 5 P$;
V16. $\neg(S 2 P \wedge S 3 P)$;
V7. $S 1 P \supset P 1 S$;
V17. $\neg(S 2 P \wedge S 4 P)$;
V8. $S 2 P \supset P 3 S$;
V18. $\neg(S 1 P \wedge S 5 P)$;
V9. $S 3 P \supset P 2 S$;
V19. $\neg(S 4 P \wedge S 5 P)$;
V10. $S 4 P \supset P 4 S$;
V20. $S 1 P \vee S 2 P \vee S 3 P \vee S 4 P \vee S 5 P$.

Единственное правило вывода в $\mathbf{C 4 V}$ - modus ponens. 
Там же был задан следующий перевод $v_{1}$ из множества формул языка силлогистики Венна $\left(\mathbf{L}_{\mathbf{V}}\right)$ в множество формул языка традиционной силлогистики $\left(\mathbf{L}_{\mathbf{T}}\right)$ :

$$
\begin{array}{ll}
v_{1}(S 1 P)=S a P \wedge P a S ; & v_{1}(S 4 P)=S i P \wedge S o P \wedge P o S ; \\
v_{1}(S 2 P)=S a P \wedge P o S ; & v_{1}(S 5 P)=S e P ; \\
v_{1}(S 3 P)=S o P \wedge P a S ; & v_{1}(\neg A)=\neg v_{1}(A) ; \\
v_{1}(A \nabla B)=v_{1}(A) \nabla v_{1}(B), & \text { где } \nabla-\text { бинарная связка. }
\end{array}
$$

Была доказана следующая метатеорема о погружаемости C4V в C4 посредством перевода $v_{1}$ :

Утверждение 2. Для любой формуль $A \in \mathbf{L}_{\mathbf{V}}$ верно, что $A$ доказуема в исчислении $\mathbf{C 4 V}$, е.т.е. $v_{1}(A)$ доказуема в $\mathbf{C 4}$.

Продемонстрируем справедливость семантического аналога последнего утверждения.

Теорема 4. Для любой формулы $A \in \mathbf{L}_{\mathbf{V}}$ верно, что $A$ является $\boldsymbol{V}$-общезначимой, е.т.е. формула $v_{1}(A)$ является $\mathbf{T}$-общезначимой.

Доказательство. Достаточно показать, что условия $\mathbf{V}$-значимости произвольной формулы $A \in \mathbf{L}_{\mathbf{V}}$ и условия $\mathbf{T}$-значимости ее перевода $v_{1}(A)$ совпадают. Используем индукцию по количеству пропозициональных связок в формуле $A \in \mathbf{L}_{\mathbf{V}}$.

Базис индукции включает пять случаев.

(1) $A=S 1 P$.

$\mathcal{V}(S 1 P, \mathbf{D}, \varphi)$, е.т.е. (в силу $\left.\mathcal{V}_{1}\right) \varphi(S)=\varphi(P)$, е.т.е. (по определению равенства множеств) $\varphi(S) \subseteq \varphi(P)$ и $\varphi(P) \subseteq \varphi(S)$, е.т.е. (в силу $\left.\mathcal{V}_{a}\right) \mathcal{V}(S a P, \mathbf{D}, \varphi)$ и $\mathcal{V}(P a S, \mathbf{D}, \varphi)$, е.т.е. (в силу $\left.\mathcal{V}_{C}\right) \mathcal{V}(S a P \wedge P a S, \mathbf{D}, \varphi)$, е.т.е. (по определению $\left.v_{1}\right) \mathcal{V}\left(v_{1}(S 1 P), \mathbf{D}, \varphi\right)$.

(2) $A=S 2 P$.

$\mathcal{V}(S 2 P, \mathbf{D}, \varphi)$, е.т.е. (в силу $\left.\mathcal{V}_{2}\right) \varphi(S) \subset \varphi(P)$, е.т.е. (по определению $\subset$ ) $\varphi(S) \subseteq \varphi(P)$ и $\varphi(P) \backslash \varphi(S) \neq \varnothing$, е.т.е. (в силу $\mathcal{V}_{a}$ и $\left.\mathcal{V}_{o}\right) \mathcal{V}(S a P, \mathbf{D}, \varphi)$ и $\mathcal{V}(P o S, \mathbf{D}, \varphi)$, е.т.е. (в силу $\left.\mathcal{V}_{C}\right) \mathcal{V}(S a P \wedge P o S, \mathbf{D}, \varphi)$, е.т.е. (по определению $\left.v_{1}\right) \mathcal{V}\left(v_{1}(S 2 P), \mathbf{D}, \varphi\right)$.

(3) $A=S 3 P$.

Рассматривается аналогично предыдущему случаю.

(4) $A=S 4 P$.

$\mathcal{V}(S 4 P, \mathbf{D}, \varphi)$, е.т.е. (в силу $\left.\mathcal{V}_{4}\right) \varphi(S) \cap \varphi(P) \neq \varnothing$ и $\varphi(S) \backslash \varphi(P) \neq \varnothing$ и $\varphi(P) \backslash \varphi(S) \neq \varnothing$, е.т.е. (в силу $\mathcal{V}_{i}$ и $\left.\mathcal{V}_{o}\right) \mathcal{V}(\operatorname{SiP}, \mathbf{D}, \varphi)$ и $\mathcal{V}(S o P, \mathbf{D}, \varphi)$ 
и $\mathcal{V}(P o S, \mathbf{D}, \varphi)$, е.т.е. (в силу $\left.\mathcal{V}_{C}\right) \mathcal{V}(S i P \wedge S o P \wedge P o S, \mathbf{D}, \varphi)$, е.т.е. (по определению $\left.v_{1}\right) \mathcal{V}\left(v_{1}(S 4 P), \mathbf{D}, \varphi\right)$.

(5) $A=S 5 P$.

$\mathcal{V}(S 5 P, \mathbf{D}, \varphi)$, е.т.е. (в силу $\left.\mathcal{V}_{5}\right) \varphi(S) \cap \varphi(P)=\varnothing$, е.т.е (в силу $\mathcal{V}_{e}$ ) $\mathcal{V}(S e P, \mathbf{D}, \varphi)$, е.т.е. (по определению $\left.v_{1}\right) \mathcal{V}\left(v_{1}(S 5 P), \mathbf{D}, \varphi\right)$.

Доказательство индуктивного перехода тривиально.

Докажем далее, что исчисление $\mathbf{C 4 V}$ адекватно формализует сформулированную выше семантику локального силлогистического языка с исходными константами 1, 2, 3, 4, 5 .

Теорема 5. Для любой формуль $A \in \mathbf{L}_{\mathbf{V}}$ верно, что $A$ доказуема в $\mathbf{C 4 V}$, е.т.е. А является $\boldsymbol{V}$-общезначимой.

Доказательство. Рассмотрим произвольную формулу $A \in \mathbf{L}_{\mathbf{V}}$. Согласно Утверждению 2 , $A$ доказуема в $\mathbf{C} 4 \mathbf{V}$, е.т.е. $v_{1}(A)$ доказуема в $\mathbf{C 4}$. Формула $v_{1}(A)$ является формулой языка традиционной силлогистики (принадлежит $\mathbf{L}_{\mathbf{T}}$ ). Поэтому, в силу Утверждения $\mathbf{1}, v_{1}(A)$ доказуема в $\mathbf{C 4 ,}$ е.т.е. $v_{1}(A)$ T-общезначима. Согласно Теореме $4, v_{1}(A)$ T-общезначима, е.т.е. формула $A$ является $\mathbf{V}$-общезначимой. Следовательно, произвольная формула $A \in \mathbf{L}_{\mathbf{V}}$ доказуема в исчислении $\mathbf{C} 4 \mathbf{V}$, е.т.е. она является $\mathbf{V}$-общезначимой.

Сформулируем исчисление в языке, включающем все силлогистические константы, и докажем его непротиворечивость и полноту относительно семантики универсального силлогистического языка.

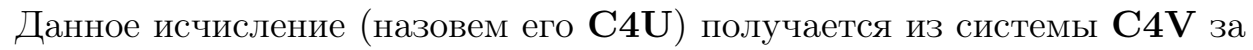
счет добавления двух новых схем аксиом:

$$
\begin{aligned}
& \text { V21. } \neg S P \text {; } \\
& \text { V22. } S x_{1} x_{2} \ldots x_{n} P \equiv\left(S x_{1} P \vee S x_{2} P \vee \ldots \vee S x_{n} P\right) \text {. }
\end{aligned}
$$

Понятие доказательства в $\mathbf{C 4 U}$ обычное.

Покажем, что множество теорем $\mathbf{C 4 U}$ и множество $\mathbf{U}$-общезначимых формул равны.

Теорема 6. Для любой формуль $A \in \mathbf{L}_{\mathbf{U}}$ верно, что если $A$ доказуема в $\mathbf{C 4 \mathbf { U } , \text { то А является } \mathbf { U } - о б щ е з н а ч и м о и ̆ . ~}$

Доказателъство. Метатеорема о семантической непротиворечивости доказывается стандартным способом. Демонстрируется, что аксиомы всех типов $\mathbf{U}$-общезначимы, а единственное правило вывода (modus ponens) coxpaняет свойство «быть U-общезначимой формулой». 
Для доказательства обратной метатеоремы предварительно обоснуем ряд вспомогательных утверждений.

Для системы C4U можно доказать синтаксический аналог метатеоремы об эквивалентной замене (Теоремы 1 ).

Теорема 7. Пусть $A$ и $B-$ произвольные формуль из $\mathbf{L}_{\mathbf{U}}, C_{A}-$ формула

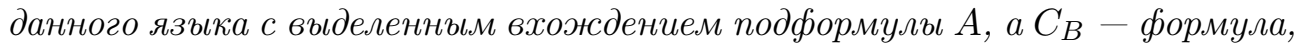
получающаяся в результате замены выделенного вхождения $A$ в $C_{A}$ на бормулу $B$. Если формула $A \equiv B$ доказуема в исчислении C4U, то и формула $C_{A} \equiv C_{B}$ доказуема в нем.

Доказательство. Исчисление $\mathbf{C 4 U}$ строится на базе классического исчисления высказываний. Языки этих двух систем отличаются типами простых формул, а сложные формулы определяются одинаково. Поэтому доказательство данной метатеоремы для $\mathbf{C 4} \mathbf{U}$ практически повторяет известное доказательство для классического исчисления высказываний.

Пусть $C$ - произвольная формула из $\mathbf{L}_{\mathbf{U}}$. Под $C^{+}$будем понимать результат замены в $C$ всех атомарных подформул вида $S P$ на $S 2 S \wedge P 2 P$, а всех атомарных подформул вида $S x_{1} x_{2} \ldots x_{n} P$ на $S x_{1} P \vee S x_{2} P \vee \ldots \vee$ $S x_{n} P$. При этом преобразовании из исходной формулы удаляются нулькомпонентная константа и все многокомпонентные константы. В составе $C^{+}$ остаются лишь однокомпонентные константы. Таким образом, $C^{+} \in \mathbf{L}_{\mathbf{V}}$ (оставаясь, конечно, элементом более широкого множества $\mathbf{L}_{\mathbf{U}}$ ).

Докажем две леммы об эквивалентности $C$ и $C^{+}$как в семантике универсального силлогистического языка, так и в исчислении $\mathbf{C 4 U}$.

Лемма 1. Для любой формуль $C \in \mathbf{L}_{\mathbf{U}}$ верно, что $C$ и $C^{+}$эквивалентнъ в семантике универсального силлогистического языка (m. е. $\mathcal{V}(C, \mathbf{D}, \varphi)$, е.т.е. $\mathcal{V}\left(C^{+}, \mathbf{D}, \varphi\right)$ в любой модели $\left.\langle\mathbf{D}, \varphi\rangle\right)$.

Доказательство. Сначала покажем, что каждая заменяемая подформула в $C$ преобразуется в формулу, эквивалентную ей в семантике универсального силлогистического языка.

Формулы вида $S P$ не являются выполнимыми. Таковыми же будут и формулы вида $S 2 S \wedge P 2 P$, поскольку они значимы в модели $\langle\mathbf{D}, \varphi\rangle$, е.т.е. $\varphi(S) \subset \varphi(S)$ и $\varphi(P) \subset \varphi(P)$, а последнее неверно ни в какой модели. Поэтому $S P$ эквивалентна $S 2 S \wedge P 2 P$.

Любая формула вида $S x_{1} x_{2} \ldots x_{n} P$ эквивалентна в семантике универсального силлогистического языка формуле $S x_{1} P \vee S x_{2} P \vee \ldots \vee S x_{n} P$ в силу условия $\mathcal{V}_{M}$ и условия значимости дизъюнктивных формул.

Для демонстрации семантической эквивалентности $C$ и $C^{+}$необходимое число раз применяем Теорему 1 
Лемма 2. Для любой формуль $C \in \mathbf{L}_{\mathbf{U}}$ верно, что формула $C \equiv C^{+}$

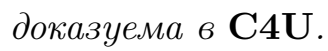

Доказателъство. Сначала нужно убедиться в том, что формулы видов $S P \equiv(S 2 S \wedge P 2 P)$ и $S x_{1} x_{2} \ldots x_{n} P \equiv S x_{1} P \vee S x_{2} P \vee \ldots \vee S x_{n} P$ доказуемы в исчислении $\mathbf{C 4 U}$.

Формулы второго типа являются аксиомами этой системы (V22).

Построим в $\mathbf{C} 4 \mathbf{U}$ доказательство формул первого типа $-S P \equiv(S 2 S \wedge$ $P 2 P$ ) (шаги, осуществляемые по производным в исчислении высказываний правилам вывода, сопровождаем в анализе отметкой «ЛВ»):

$\begin{array}{ll}\text { 1. } S 1 S ; & \text { V12 } \\ \text { 2. } \neg(S 1 S \wedge S 2 S) & \text { V13 } \\ \text { 3. } \neg S 2 S & 1,2 ; \text { ЛВ } \\ \text { 4. } \neg(S 2 S \wedge P 2 P) & 3 ; \text { ЛВ } \\ \text { 5. } \neg S P & \text { V21 } \\ \text { 6. } S P \equiv(S 2 S \wedge P 2 P) & 4,5 ; \text { ЛВ. }\end{array}$

Далее, используя нужное число раз Теорему 7, приходим к выводу о том, что формула $C \equiv C^{+}$доказуема в $\mathbf{C} 4 \mathbf{U}$.

Докажем, наконец, полноту системы $\mathbf{C 4} \mathbf{U}$ относительно семантики универсального силлогистического языка.

Теорема 8. Для любой формуль $A \in \mathbf{L}_{\mathbf{U}}$ верно, что если $A$ является

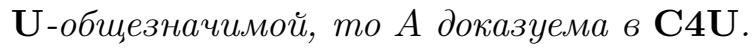

Доказателъство. Рассмотрим произвольную формулу $A \in \mathbf{L}_{\mathbf{U}}$. Допустим, что она является $\mathbf{U}$-общезначимой.

Согласно Лемме 1. формула $A$ эквивалентна в семантике универсального силлогистического языка формуле $A^{+}$. Значит, и $A^{+}$является $\mathrm{U}$-общезначимой.

Но формула $A^{+} \in \mathbf{L}_{\mathbf{V}}$, поскольку в ней содержатся только однокомпонентные константы. Условия значимости таких формул в семантике универсального языка и в семантике локального языка с исходными константами $1,2,3,4,5$ совпадают. Следовательно, формула $A^{+}$является $\mathrm{V}$-общезначимой.

Тогда, в силу Теоремы $5, A^{+}$доказуема в исчислении $\mathbf{C 4 V}$. Но $\mathbf{C 4 V}$ является подсистемой $\mathbf{C 4} \mathbf{U}$, поэтому формула $A^{+}$доказуема и в исчислении $\mathbf{C} 4 \mathbf{U}$.

Согласно Лемме 2 , формула $A \equiv A^{+}$доказуема в $\mathbf{C} 4 \mathbf{U}$. Но поскольку и $A^{+}$является теоремой этой системы, формула $A$ доказуема в $\mathbf{C} 4 \mathbf{U}$. 


\section{5. Заключение}

Таким образом, мы построили язык позитивной силлогистики, в алфавите которого содержатся все силлогистические константы, трактуемые как знаки отношений между двумя непустыми множествами. Была сформулирована точная семантика для этого языка и осуществлена ее формализация. Средствами предложенной логической теории предстот решить ряд важных для общего понимания силлогистики вопросов, в частности, установить критерии полноты наборов силлогистических констант.

Одним из возможных направлений развития данного подхода является отказ от требования непустоты субъектов и предикатов категорических высказываний. В этом случае к пяти примитивным, эйлеровским отношениям между двумя непустыми множествами добавятся еще три: объемные отношения между двумя пустыми терминами, между пустым субъектом и непустым предикатом, между непустым субъектом и пустым предикатом. Появится возможность исследовать такие системы позитивной силлогистики в «локальных» языках, которые не являются дефинициально эквивалентными традиционной (системе $\mathbf{C 4}$ ).

\section{Литература}

Васильев, 1910 - Васильев Н.А. О частных суждениях, о треугольнике противоположностей, о законе исключенного четвертого // Ученые записки Казанского университета. Год 77, 1910. Кн. 10. С. 1-47.

Дубаков, Маркин, 2007 - Дубаков Д.В., Маркин В.И. Система силлогистики с исходными константами, соответствующими круговым диаграммам // Труды научно-исследовательского семинара Логического центра Института философии РАН. Вып. ХVIII. М.: ИФ РАН, 2007. С. 63-75.

Лукасевич, 1959 - Лукасевич Я. Аристотелевская силлогистика с точки зрения современной формальной логики. М.: Изд-во иностр. лит-ры, 1959. 313 с.

Мчедлишвили, 1985 - Мчедлишвили Л.И. Реконструкция метода эктезиса и системы позитивной силлогисики // Логика Аристотеля. Материалы симпозиума. Тбилиси: Изд-во Тбилисского ун-та, 1985. С. 21-35.

Смирнов, 2002 - Смирнов В.А. Логические методы анализа научного знания. М.: Эдиториал УРСС, 2002. 263 с.

Venn, 1881 - VennJ. Symbolic Logic. London: Macmillan and Co., 1881. 446 p. 


\title{
VLADIMIR I. MARKIN \\ Syllogistic as the logic of all relations between two non-empty sets
}

\author{
Vladimir I. Markin \\ Lomonosov Moscow State University, \\ 27/4 Lomonosovskiy prospect, Moscow, 119991, Russian Federation. \\ E-mail: markin@philos.msu.ru
}

\begin{abstract}
In the paper we offer the language of positive syllogistic the alphabet of which contains all syllogistic constants. These constants are interpreted as the signs of different relationships between two non-empty sets (the extensions of general terms). Among these relationships we give accent to the relationships corresponding only to one from five EulerGergonne diagrams: (1) equality, (2) strict inclusion of the first set into the second, (3) strict inclusion of the second set into the first, (4) overlap, (5) exclusion. The other relationships can be represented as various combinations of Eulerian. Every constant $k$ in the 'universal' syllogistic language is encoded with a sequence of numbers from 1 to 5 in accordance with the diagrams where the proposition of the form $S k P$ is true. For example, the constant 12 represents the inclusion relationship of one non-empty set into another, the constant 1234 represents compatibility relationship between two non-empty sets. We formulate the set theoretical semantics based on the intuitive interpretation of syllogistic constants for this 'universal' syllogistic language. We introduce the notion of the definability of a syllogistic constant in a 'local' syllogistic language that contains only some of such constants, and the notion of the completeness of a set of initial constants in such a language. A constant $k$ is definable in a 'local' syllogistic language iff in the language there exists a formula $A$ that contains exactly two general terms $S$ and $P$, and $A$ is equivalent to the formula $S k P$ in the semantics of the 'universal' syllogistic language. A set of syllogistic constants $\left\{k_{1}\right.$, $\left.k_{2}, \ldots, k_{m}\right\}$ is complete iff any syllogistic constant is definable in the 'local' language with the initial constants $k_{1}, k_{2}, \ldots, k_{m}$. As an example, we demonstrate that the sets of the initial constants of traditional syllogistic $(a, e, i, o)$ and Venn's syllogistic (the signs of five Eulerian relationships) are complete. We construct the axiomatic calculus in the 'universal' syllogistic language and prove soundness and completeness metatheorems.
\end{abstract}

Keywords: syllogistic, syllogistic constant, formal language, semantics, definability, complete set of constants, axiomatic calcululs, soundness and completeness metatheorems

For citation: Markin V.I. "Sillogistika kak logika vsekh otnoshenii mezhdu dvumya nepustymi mnozhestvami" [Syllogistic as the logic of all relations between two non-empty sets], Logicheskie Issledovaniya / Logical Investigations, 2020, Vol. 26, No. 2, pp. 39-57. DOI: 10.21146/2074-1472-2020-26-2-39-57 (In Russian) 


\section{References}

Vasiliev, 1910 - Vasiliev N.A. "O chastnyh suzhdeniyah, o treugol'nike protivopolozhnostej, o zakone isklyuchennogo chetvertogo" [On particular statements, on the triangle of opposition, on the law of excluded forth], in: Uchenye zapiski Kazanskogo universiteta Proceedings of Kazan University. Year 77, 1910. Vol. 10, pp. 1-47. (In Russian)

Dubakov, Markin, 2007 - Dubakov, D.V., Markin, V.I. "Sistema sillogistiki s ishodnymi konstantami, sootvetstvuyushimi krugovym diagrammam" [The syllogistic system with initial constants corresponding to circular diagrams], in: Trudy nauchno-issledovatelskogo seminara Logicheskogo centra Instituta filosofii RAN [Proceedings of scientific research seminar of Logical center Institute of Philosophy RAS ]. Vol. XVIII. Moscow: Institute of Philosophy RAS, 2007, pp. 63-75. (In Russian)

Łukasiewicz, 1959 - Łukasiewicz, J. Aristotelevskaya sillogistika s tochki zreniya sovremennoj formal'noj logiki [Aristotile's sillogistic from the standpoint of modern formal logic]. Moscow: Foreign Literature Publ., 1959. 313 pp. (In Russian)

Mchedlishvili, 1985 - Mchedlishvili L.I. "Rekonstrukciya metoda ektezisa i sistemy pozitivnoj sillogisiki" [Reconstruction of ecthesis method and systems of positive syllogistic], in: Logika Aristotelya. Materialy simpoziuma [Aristotle's logic. Proceedings of symposium]. Tbilisi: Tbilisi University Publ., 1985, pp. 21-35. (In Russian)

Smirnov, 2002 - Smirnov, V.A. Logicheskie metody analiza nauchnogo znaniya [Logical methods of the analyses of scientific knowledge]. Moscow: Editorial URSS, 2002. 263 pp. (In Russian)

Venn, 1881 - Venn, J. Symbolic Logic. London: Macmillan and Co., 1881. 446 pp. 